\title{
Broken symmetries and magnetic dynamos
}

\author{
John V. Shebalin \\ Astromaterials Research E Exploration Science Office \\ NASA Johnson Space Center, Houston, Texas 77058
}

\begin{abstract}
Phase space symmetries inherent in the statistical theory of ideal magnetohydrodynamic (MHD) turbulence are known to be broken dynamically to produce large-scale coherent magnetic structure. Here, results of a numerical study of decaying MHD turbulence are presented that show large-scale coherent structure also arises and persists in the presence of dissipation. Dynamically broken symmetries in MHD turbulence may thus play a fundamental role in the dynamo process.
\end{abstract}

PACS: 47.20.Ky, 47.27.-i, 91.25.Cw, 95.30.Qd

Keywords: Symmetry breaking, Turbulence, Coherent Structures, Magnetic Dynamo

\section{Introduction}

We examine (both ideal and decaying) incompressible, homogeneous, three-dimensional (3-D) magnetohydrodynamic (MHD) turbulence using Fourier spectral methods. In these methods, physical fields such as the turbulent velocity $\mathbf{u}$ and magnetic induction $\mathbf{b}$ are represented by truncated Fourier series on an evenly spaced $N^{3}$ computational grid:

$$
\mathbf{u}(\mathbf{x})=\frac{1}{N^{3 / 2}} \sum_{0<|\mathbf{k}| \leq K} \tilde{\mathbf{u}}(\mathbf{k}) e^{i \mathbf{k} \cdot \mathbf{x}}, \quad \mathbf{b}(\mathbf{x})=\frac{1}{N^{3 / 2}} \sum_{0<|\mathbf{k}| \leq K} \tilde{\mathbf{b}}(\mathbf{k}) e^{i \mathbf{k} \cdot \mathbf{x}} .
$$

The wave vectors $\mathbf{k}$ in $\mathrm{k}$-space have integer components between $-N / 2$ and $+N / 2$, while the corresponding discrete set of position vectors $\mathbf{x}$ in $\mathrm{x}$-space have components $x_{j}=2 \pi m_{j} / N\left(j=1,2,3 ; 0 \leq m_{j}<N\right)$, where $N$ is the number of grid points in each dimension. The 'isotropic truncation' radius is $K$, with $0<k \leq K<N / 2$ and $k=|\mathbf{k}|$, so that retained coefficients fit within a sphere in $\mathrm{k}$-space; the exact value of $K$ is set by de-aliasing requirements ${ }^{1}$.

Fourier spectral methods place MHD turbulence in a periodic box that can represent either a small volume interior to a larger volume of plasma, or a complete mass of plasma within a 3 -torus (such as the universe). Also, isolated plasma (such as a star) may be thought of as residing in a box large enough so that any plasma flows or magnetic fields outside of or on the surface of the box have negligible energy. In this later case, a periodic box serves as an approximate surrogate of the more complicated physical situation. Thus, Fourier models are of value by providing at least qualitative information about laboratory, geophysical and astrophysical turbulence, especially if dissipation is included.

Statistical studies of such Fourier models were initiated by T. D. Lee, in a prescient paper ${ }^{2}$ that demonstrated the existence of canonical ensembles based on the ideal invariants in the phase space defined by the set of independent Fourier modes. The total energy $E$ is one ideal invariant, and two other invariants were soon discovered: $H_{M}$, the magnetic helicity ${ }^{3}$ and $H_{C}$, the cross helicity ${ }^{4}$. Once computational resources were sufficient, basic aspects of the 'absolute equilibrium ensemble theory' of 3-D MHD turbulence were described $^{5}$. The broken symmetry and non-ergodicity contained in this theory when magnetic and cross helicity are non-zero were subsequently recognized ${ }^{6,7,8,9}$.

Early computational studies of dissipative, helical MHD turbulence, motivated by the dynamo problem, found evidence of an inverse cascade of magnetic helicity from small scales to large ones, with a concomitant generation of strong, large-scale magnetic fields ${ }^{10,11}$. The presence of helicity is critical to this process, as 
studies of non-helical MHD turbulence have found only small-scale dynamo action ${ }^{12}$. Broken symmetry and non-ergodicity, in addition to their intrinsic interest, appear relevant to theoretically understanding the magnetic dynamo, and this is our focus here. We will build on previous results in ideal MHD turbulence and examine their relevance to dissipative MHD turbulence.

A recent paper ${ }^{13}$ examined ideal, homogeneous, incompressible, 3-D MHD turbulence in a rotating frame of reference with and without a mean magnetic field. There turn out to be five general cases of incompressible, homogeneous 3-D MHD turbulence, categorized by the absence or presence of a mean magnetic field $\mathbf{B}_{\mathrm{o}}$ and/or an overall system rotation $\boldsymbol{\Omega}_{\mathrm{o}}$; these cases are shown in Table 1 . (Here, the cases are slightly reordered.) In Table 1, a new invariant, the 'parallel helicity' $H_{P} \equiv H_{C}-\sigma H_{M}$ is indicated, which appears when $\boldsymbol{\Omega}_{\mathrm{o}}=\sigma \mathbf{B}_{\mathrm{o}} \neq 0$ ( $\sigma$ is a non-zero real number). In the recent study, very long-time $32^{3}$ simulations again demonstrated that ensemble predictions for ideal MHD turbulence were dynamically broken to produce energetic large-scale coherent structures, both with and without overall rotation.

In the present work, dissipation is introduced through non-zero viscosity $\nu$ in eq. (2) and magnetic diffusivity $\eta$ in eq. (3) (see below). Results from long-time non-rotating and rotating $64^{3}$ simulations are presented and large-scale coherent structures, as seen in previous ideal runs, are still present. In effect, this is a 'magnetic dynamo process' that is inherent to MHD turbulence, a process that requires an initial energetic stirring of a magneto-fluid, but does not require any continuous external forcing, such as convection (which can, nevertheless, provide a stirring mechanism). A magnetic dynamo may thus be ultimately related to dynamically broken symmetry inherent within MHD turbulence.

\section{Basic Theory}

The non-dimensional form of the incompressible MHD equations in a rotating frame of reference with constant angular velocity $\boldsymbol{\Omega}_{\mathrm{o}}$ and a mean (i.e., uniform and constant) magnetic induction $\mathbf{B}_{\mathrm{o}}$ are well known ${ }^{14}$. They are

$$
\begin{aligned}
\frac{\partial \boldsymbol{\omega}}{\partial t} & =\nabla \times\left[\mathbf{u} \times\left(\boldsymbol{\omega}+2 \boldsymbol{\Omega}_{\mathrm{o}}\right)+\mathbf{j} \times\left(\mathbf{b}+\mathbf{B}_{\mathrm{o}}\right)\right]+\nu \nabla^{2} \boldsymbol{\omega} \\
\frac{\partial \mathbf{b}}{\partial t} & =\nabla \times\left[\mathbf{u} \times\left(\mathbf{b}+\mathbf{B}_{\mathrm{o}}\right)\right]+\eta \nabla^{2} \mathbf{b} .
\end{aligned}
$$

Here, the turbulent velocity $\mathbf{u}$ and magnetic induction $\mathbf{b}$ satisfy $\nabla \cdot \mathbf{u}=\nabla \cdot \mathbf{b}=0$, and the associated vorticity $\boldsymbol{\omega}$ and electric current $\mathbf{j}$ are $\boldsymbol{\omega}=\nabla \times \mathbf{u}$ and $\mathbf{j}=\nabla \times \mathbf{b}$ ). Density does not appear because it equals unity; again, $\nu$ is the kinematic viscosity and $\eta$ is the magnetic diffusivity.

In terms of the Fourier expansions (1), $\mathrm{x}$-space $\nabla \rightarrow i \mathbf{k}$ in $\mathrm{k}$-space and

$$
\tilde{\boldsymbol{\omega}}(\mathbf{k})=i \mathbf{k} \times \tilde{\mathbf{u}}(\mathbf{k}), \quad \tilde{\mathbf{j}}(\mathbf{k})=i \mathbf{k} \times \tilde{\mathbf{b}}(\mathbf{k}), \quad \mathbf{k} \cdot \tilde{\mathbf{u}}(\mathbf{k})=\mathbf{k} \cdot \tilde{\mathbf{b}}(\mathbf{k})=0 .
$$

There are two independent complex components in a vector Fourier coefficient, for a total of four real and imaginary parts for each Fourier mode $\tilde{\mathbf{u}}(\mathbf{k})$ and $\tilde{\mathbf{b}}(\mathbf{k})$. Since the reality of $\mathbf{u}(\mathbf{x})$ requires that $\tilde{\mathbf{u}}(\mathbf{k})=\tilde{\mathbf{u}}^{*}(-\mathbf{k})$, etc., where ${ }^{*}$ denotes complex conjugation, the number of independent modes is one half of the total number $\mathcal{N} \approx 4 \pi K^{3} / 3$ of non-zero $\mathbf{k}$ within the ball $k \leq K$. The number of independent $\mathbf{k}$ is thus $\mathcal{N}^{\prime}=\frac{1}{2} \mathcal{N}$ and the dimension of the phase space $\Gamma$ of independent real and imaginary Fourier coefficients is $N_{\Gamma}=4 \mathcal{N}$. For the $64^{3}$ grid numerical results to be presented here, $\mathcal{N}^{\prime}=57656$ and $N_{\Gamma}=461248$.

If we set $\nu=0$ in (2) and $\eta=0$ in (3), we obtain the equations of ideal MHD turbulence. While any real flows must have $\nu \neq 0$ and $\eta \neq 0$, a study of ideal turbulence produces some interesting theoretical results that help in understanding aspects of real turbulence, particularly at larger scales of the flow where dissipation is minimal. Next, we review ideal MHD turbulence and then discuss real MHD turbulence. 


\begin{tabular}{cccc}
\hline Case & Mean Field & Rotation & Invariants \\
\hline I & $\mathbf{B}_{\mathrm{o}}=0$ & $\boldsymbol{\Omega}_{\mathrm{o}}=0$ & $E, H_{C}, H_{M}$ \\
II & $\mathbf{B}_{\mathrm{o}} \neq 0$ & $\boldsymbol{\Omega}_{\mathrm{o}}=0$ & $E, H_{C}$ \\
III & $\mathbf{B}_{\mathrm{o}}=0$ & $\boldsymbol{\Omega}_{\mathrm{o}} \neq 0$ & $E, H_{M}$ \\
IV & $\mathbf{B}_{\mathrm{o}} \neq 0$ & $\boldsymbol{\Omega}_{\mathrm{o}}=\sigma \mathbf{B}_{\mathrm{o}}$ & $E, H_{P}$ \\
V & $\mathbf{B}_{\mathrm{o}} \neq 0$ & $\boldsymbol{\Omega}_{\mathrm{o}} \times \mathbf{B}_{\mathrm{o}} \neq 0$ & $E$ \\
\hline
\end{tabular}

Table 1: Invariants for ideal MHD turbulence.

\section{Ideal MHD Turbulence}

First, we define the volume average (i.e., the average value per grid point) of a product $\phi(\mathbf{x}) \psi(\mathbf{x})$ in a periodic box $\mathcal{B}$ of side length $2 \pi$ as

$$
[\phi \psi] \equiv \frac{1}{(2 \pi)^{3}} \int_{\mathcal{B}} \phi(\mathbf{x}) \psi(\mathbf{x}) d^{3} x=\frac{1}{N^{3}} \sum_{0<|\mathbf{k}| \leq K} \tilde{\phi}(\mathbf{k}) \tilde{\psi}^{*}(\mathbf{k}) .
$$

Note that the sum over $\mathbf{k}$ counts each independent contribution twice, since $\tilde{\mathbf{u}}(-\mathbf{k})=\tilde{\mathbf{u}}^{*}(\mathbf{k})$. Since all functions are periodic, we can use equations (1) through (5), along with integration by parts to derive the following relations ${ }^{13,15}$ :

$$
\begin{aligned}
\frac{d E}{d t} & =-2(\nu \Omega+\eta J), \\
\frac{d H_{C}}{d t} & =\boldsymbol{\Omega}_{\mathrm{o}} \cdot[\mathbf{b} \times \mathbf{u}]-\frac{1}{2}(\nu+\eta)[\mathbf{j} \cdot \boldsymbol{\omega}], \\
\frac{d H_{M}}{d t} & =\mathbf{B}_{\mathrm{o}} \cdot[\mathbf{b} \times \mathbf{u}]-\eta[\mathbf{j} \cdot \mathbf{b}] .
\end{aligned}
$$

Above, we have the (volume-averaged) energy $E$, enstrophy $\Omega$, mean-squared current $J$, cross helicity $H_{C}$ and magnetic helicity $H_{M}$ (here, $\mathbf{a}$ is defined by $\mathbf{b}=\nabla \times \mathbf{a}$ with $\left.\nabla \cdot \mathbf{a}=0\right)$ :

$$
E=\frac{1}{2}\left[|\mathbf{u}|^{2}+|\mathbf{b}|^{2}\right], \quad \Omega=\frac{1}{2}\left[|\boldsymbol{\omega}|^{2}\right], \quad J=\frac{1}{2}\left[|\mathbf{j}|^{2}\right], \quad H_{C}=\frac{1}{2}[\mathbf{u} \cdot \mathbf{b}], \quad H_{M}=\frac{1}{2}[\mathbf{a} \cdot \mathbf{b}] .
$$

At this point we note that if $\boldsymbol{\Omega}_{\mathrm{o}}=\sigma \mathbf{B}_{\mathrm{o}}$, i.e., if $\boldsymbol{\Omega}_{\mathrm{o}}$ and $\mathbf{B}_{\mathrm{o}}$ are non-zero and parallel, then eq. (7) can be added to $-\sigma$ times eq. (8) to yield

$$
\frac{d H_{P}}{d t}=-\frac{1}{2}(\nu+\eta)[\mathbf{j} \cdot \boldsymbol{\omega}]+\sigma \eta[\mathbf{j} \cdot \mathbf{b}], \quad H_{P} \equiv H_{C}-\sigma H_{M}=\frac{1}{2}[(\mathbf{u}-\sigma \mathbf{a}) \cdot \mathbf{b}] .
$$

The recently discovered quantity $H_{P}$ has been named the 'parallel helicity'13,16.

When $\nu=\eta=0$, equations (6), (7), (8) and (10) lead us immediately to the invariant integrals for MHD turbulence for various values of $\mathbf{B}_{\mathrm{o}}$ and $\boldsymbol{\Omega}_{\mathrm{o}}$. The five general cases of incompressible, homogeneous, $3-\mathrm{D}$ MHD turbulence appear in Table 1. We see that as a mean field or an overall rotation is imposed, or both, the number of invariants drop from three to two to one.

The probability density function $D$ of the absolute equilibrium ensemble, for Case I in Table 1, has the canonical form ${ }^{5}$

$$
D=\frac{1}{Z} \exp \left(-\alpha E-\beta H_{C}-\gamma H_{M}\right), \quad Z=\int_{\Gamma} \exp \left(-\alpha E-\beta H_{C}-\gamma H_{M}\right) d \Gamma .
$$

In eq. (11), $Z$ is the partition function and $\Gamma$ is the phase space defined by the $N_{\Gamma}$ independent Fourier coefficients. Using eqs. (5) and (9), the integrand in (11) becomes an explicit quadratic form in the modal quantities $\tilde{\mathbf{u}}(\mathbf{k})$ and $\tilde{\mathbf{b}}(\mathbf{k})$; evaluating the Gaussian integral gives the partition function $Z$ :

$$
Z=\prod_{0<|\mathbf{k}| \leq K} Z(\mathbf{k}), \quad Z(\mathbf{k})=\frac{\pi^{4}}{\left(\delta^{4}-\alpha^{2} \gamma^{2} / k^{2}\right)^{2}}, \quad \delta^{2}=\alpha^{2}-\beta^{2} / 4 .
$$


In arriving at the above, it is necessary that $\alpha>0$ and $\delta^{2}>\alpha|\gamma| / k>0$. Also, the product is over the independent $\mathbf{k}$, i.e., $\mathbf{k}$ but not $-\mathbf{k}$. Therefore, we have $\mathcal{N}^{\prime}=\frac{1}{2} \mathcal{N}$ factors $Z(\mathbf{k})$ in eq. (12).

Using (11), canonical ensemble predictions for moments of the $\tilde{\mathbf{u}}(\mathbf{k})$ and $\tilde{\mathbf{b}}(\mathbf{k})$ can be made ${ }^{5}$ for Case I in Table 1. In what follows, the ensemble prediction of a quantity $Q$ will be denoted by $\langle Q\rangle$ and the time-average by $\bar{Q}$, where

$$
\langle Q\rangle=\int_{\Gamma} Q D d \Gamma, \quad \bar{Q}=\frac{1}{T_{f}} \int_{0}^{T_{f}} Q d t
$$

The predicted first-order moments are $\langle\tilde{\mathbf{u}}(\mathbf{k})\rangle=\langle\tilde{\mathbf{b}}(\mathbf{k})\rangle=0$ for all cases. The ensemble predictions for second-order moments for Case I in Table 1 are given in the following equations (again, $\delta^{2}=\alpha^{2}-\beta^{2} / 4$ ):

$$
\begin{aligned}
& \text { (a) }\left\langle|\tilde{\mathbf{u}}(\mathbf{k})|^{2}\right\rangle=\frac{2 \alpha\left(\delta^{2}-\gamma^{2} / k^{2}\right)}{\delta^{4}-\alpha^{2} \gamma^{2} / k^{2}}, \quad \text { (c) }\left\langle\tilde{\mathbf{u}}(\mathbf{k}) \cdot \tilde{\mathbf{b}}^{*}(\mathbf{k})\right\rangle=\frac{-\beta \delta^{2}}{\delta^{4}-\alpha^{2} \gamma^{2} / k^{2}} \\
& \text { (b) }\left\langle|\tilde{\mathbf{b}}(\mathbf{k})|^{2}\right\rangle=\frac{2 \alpha \delta^{2}}{\delta^{4}-\alpha^{2} \gamma^{2} / k^{2}}, \quad \text { (d) }\left\langle\tilde{\mathbf{a}}(\mathbf{k}) \cdot \tilde{\mathbf{b}}^{*}(\mathbf{k})\right\rangle=\frac{-2 \gamma \alpha^{2} / k^{2}}{\delta^{4}-\alpha^{2} \gamma^{2} / k^{2}} \text {. }
\end{aligned}
$$

In (14), modal expectation values of (a) kinetic energy, (b) magnetic energy, (c) cross helicity, and (d) magnetic helicity are given for Case I of Table 1. As for the other cases in Table 1, eq. (14) applies as follows: Case II, set $\gamma=0$; Case III, set $\beta=0$; Case IV, set $\gamma=-\sigma \beta$, so that eq. (11) becomes $D=Z^{-1} \exp \left(-\alpha E-\beta H_{P}\right) ;$ and in Case $\mathrm{V}$, set $\beta=\gamma=0$.

The modal predictions in (14) can be summed to produce the expectation values $\left\langle E_{K}\right\rangle,\left\langle E_{M}\right\rangle,\left\langle H_{C}\right\rangle$, and $\left\langle H_{M}\right\rangle$ of kinetic energy, magnetic energy, cross helicity, and magnetic helicity, respectively; the total energy is, of course, $\langle E\rangle=\left\langle E_{K}\right\rangle+\left\langle E_{M}\right\rangle$. First, since $\left\langle|\tilde{\mathbf{b}}(\mathbf{k})|^{2}\right\rangle \geq\left\langle|\tilde{\mathbf{u}}(\mathbf{k})|^{2}\right\rangle$ term by term, it is immediately apparent that $\left\langle E_{M}\right\rangle \geq\left\langle E_{K}\right\rangle$ for all cases in Table 1. Next, using eqs. (5), (9) and (14), the following algebraic relations can be derived:

$$
\begin{aligned}
\alpha\langle E\rangle+\beta\left\langle H_{C}\right\rangle+\gamma\left\langle H_{M}\right\rangle & =2 r \quad\left(r \equiv \frac{\mathcal{N}}{N^{3}}\right) \\
2 \alpha\left\langle H_{C}\right\rangle+\beta\left\langle E_{M}\right\rangle & =0 \\
\alpha\left(\langle E\rangle-2\left\langle E_{M}\right\rangle\right)-\gamma\left\langle H_{M}\right\rangle & =0 .
\end{aligned}
$$

(For the $64^{3}$ runs to be described presently, $r=0.43988$.) The inhomogeneous set of linear equations (15) can easily be solved to yield

$$
\begin{aligned}
\alpha & =\frac{r\left\langle E_{M}\right\rangle}{\left\langle E_{M}\right\rangle\left(\langle E\rangle-\left\langle E_{M}\right\rangle\right)-\left\langle H_{C}\right\rangle^{2}} \\
\beta & =-2 \frac{\left\langle H_{C}\right\rangle}{\left\langle E_{M}\right\rangle} \alpha \\
\gamma & =-\frac{2\left\langle E_{M}\right\rangle-\langle E\rangle}{\left\langle H_{M}\right\rangle} \alpha .
\end{aligned}
$$

Equations (14), (15) and (16) will be taken as definitive in that they correct various small errors appearing previously $^{7,8,15,13}$.

For the constants of the motion in eqs. (16), we will generally use $\langle E\rangle=\bar{E},\left\langle H_{C}\right\rangle=\bar{H}_{C}$ and $\left\langle H_{M}\right\rangle=$ $\bar{H}_{M}$, where the time-averages come from numerical simulations. Also, recall that $\left\langle E_{M}\right\rangle \geq\left\langle E_{K}\right\rangle$, so that $\left.2\left\langle E_{M}\right\rangle-\langle E\rangle\right) \geq 0$. Thus, using either eq. (14), (15) or (16), we see that we always have $\beta \bar{H}_{C} \leq 0$ and $\gamma \bar{H}_{M} \leq 0$, regardless of the signs of $\bar{H}_{C}$ and $\bar{H}_{M}$. These relations will be seen presently to have important ramifications. 
The results presented in (16) indicate that the inverse temperatures $\alpha, \beta$ and $\gamma$ are not actually independent, but all depend on one variable parameter: $\left\langle E_{M}\right\rangle$. Thus, if we wish to find the values of $\alpha, \beta$ and $\gamma$ associated with a particular set of $\langle E\rangle,\left\langle H_{C}\right\rangle$ and $\left\langle H_{M}\right\rangle$, we must find the expectation value $\left\langle E_{M}\right\rangle$. This can be done after we define the entropy of the system.

The entropy $S$ follows from the standard formula $S=-\langle D \ln D\rangle$, where $D$ is given by eq. (11). Using eqs. (11), (12) and (15), we have (the summation has $\mathcal{N}$ terms)

$$
S=2(r+\mathcal{N} \ln \pi)-\sum_{0<|\mathbf{k}| \leq K} \ln \left(\delta^{4}-\alpha^{2} \gamma^{2} / k^{2}\right) .
$$

As discussed above, the proper values of $\alpha, \beta$ and $\gamma$ in (17) are determined from eqs. (16) and depend on $\left\langle E_{M}\right\rangle$, which is not known beforehand.

Using results discussed by Khinchin ${ }^{19}$, and later applied to MHD turbulence ${ }^{6,20}$, we define an 'entropy functional' $S^{\prime}(\tau)$ by

$$
S^{\prime}(\tau)=2(r+\mathcal{N} \ln \pi)-\sum_{0<|\mathbf{k}| \leq K} \ln \left({\delta^{\prime}}^{4}-\alpha^{\prime 2} \gamma^{\prime 2} / k^{2}\right) .
$$

In the above equation, $\delta^{\prime 2}=\alpha^{\prime 2}-{\beta^{\prime}}^{2} / 4$, and $\alpha^{\prime}, \beta^{\prime}, \gamma^{\prime}$ are functions of $\tau$ in the same way as $\alpha, \beta$ and $\gamma$ in (16) are functions of $\left\langle E_{M}\right\rangle$. The functional $S^{\prime}(\tau)$ has one global minimum at $\tau=\left\langle E_{M}\right\rangle$; this minimum can found numerically to yield the entropy $S=S^{\prime}\left(\left\langle E_{M}\right\rangle\right)$.

The results in this section comprise established results which are, nevertheless, not necessarily well known. This has motivated the relatively brief and hopefully sufficient presentation given above, as well as the next section, where we discuss some novel features due to the pseudoscalar constants of the motion.

\section{Broken Ergodicity}

Now, we consider the effect of the classical symmetry transformations of charge inversion $C$, spatial reflection (or parity) $P$ and time reversal $T$. These discrete transformations have played an essential role in quantum physics $^{17}$; as it turns out, they are also essential for understanding homogeneous turbulence ${ }^{9}$. Table 2 shows the effect of $P, C$ and $T$ on various quantities in MHD turbulence. It can easily be seen that the equations of ideal MHD [(2) and (3) with $\nu=\eta=0$ ] are invariant under $P, C$ or $T$. However, the helicities $H_{C}$, $H_{M}$ and $H_{P}$ change sign under $P$ and/or $C$, but not $T$ (therefore $T$ does not appear to play a fundamental role in ideal turbulence). Also, recall that $H_{P} \equiv H_{C}-\sigma H_{M}$, where $\sigma$ is defined by $\boldsymbol{\Omega}_{\mathrm{o}}=\sigma \mathbf{B}_{\mathrm{o}}$; $\sigma$ is thus a pseudoscalar under $C$ but not $P$ or $T$.

Since $H_{C}, H_{M}$ and $H_{P}$ are pseudoscalars under $P$ and/or $C$, it is not immediately apparent that $D$ as given in eq. (11) is invariant under $P$ and $C$. However, as was mentioned above following eq. (16), the helicities and their associated inverse temperatures always satisfy $\beta\left\langle H_{C}\right\rangle \leq 0$ and $\gamma\left\langle H_{M}\right\rangle \leq 0$. Thus, the inverse temperatures $\beta$ and $\gamma$ behave in the same manner under $P, C$ and $T$ as their associated helicities. The fundamental result is that $\beta$ and $\gamma$ transform as pseudoscalars under $P$ and/or $C$, as is explicitly seen in eqs. (16). Since $\beta$ and $\gamma$ always take on a sign opposite to that of their associated helicities, $\beta\left\langle H_{C}\right\rangle$ and $\gamma\left\langle H_{M}\right\rangle$ are scalars under $P$ or $C$.

The novel result ${ }^{9}$ of all this is that the ensemble defined by the probability density function $D$ given in (11) consists of dynamically unconnected components. When $D$ is used for ensemble averaging, the integral over phase space includes these disjoint components that can be identified by 'set characteristic functions' with the form $\chi_{C}=H_{C} /\left|H_{C}\right|, \chi_{M}=H_{M} /\left|H_{M}\right|$ and $\chi_{P}=H_{P} /\left|H_{P}\right|$, depending on which case in Table 1 is under consideration. In other words, the phase space 'surface of constant energy' is a union of disjoint sets marked by $\chi_{C}= \pm 1, \chi_{M}= \pm 1$ or $\chi_{P}= \pm 1$. The number of disjoint components for the cases in Table 1 is $2^{M}$, where $M$ is the number of invariant helicities listed in each of the five cases (i.e., Case I has four components, Cases II, III and IV have two components each, and Case V has one component). The Birkhoff-Khinchin theorem ${ }^{19}$, tells us that an ensemble is ergodic if and only if the surface of constant energy has no disjoint components. Therefore, in Table 1, only Case V is ergodic, while Cases I, II, III and IV are non-ergodic.

As usual for canonical ensembles, although the expectation value (13) is integrated over all phase space, it takes its essential value only from the components of constant $E, \pm H_{C}$ and $\pm H_{M}$ (on these components, 


\begin{tabular}{lllll}
\hline & $I$ & $P$ & $C$ & $T$ \\
\hline $\mathbf{u}$ & + & - & + & - \\
$\boldsymbol{\omega}$ & + & + & + & - \\
$\mathbf{b}$ & + & + & - & - \\
$\mathbf{j}, \mathbf{a}$ & + & - & - & - \\
$H_{C}$ & + & - & - & + \\
$H_{M}$ & + & - & + & + \\
$H_{P}$ & + & - & - & + \\
\hline
\end{tabular}

Table 2: Effect of $P, C, T$ on the signs of various quantities.

$E=\bar{E}, H_{C}=\bar{H}_{C}$ and $\left.H_{M}=\bar{H}_{M}\right)$. As discussed above, the sign taken by $\beta$ and $\gamma$ on each component is as required by the conditions $\beta \bar{H}_{C} \leq 0$ and $\gamma \bar{H}_{M} \leq 0$. Thus, under $P$ and $C$, the scalars $\beta H_{C}$ and $\gamma H_{M}$ have the same non-positive value on each component, so that the statistical theory is invariant under $P$ and $C$ (and $T$ ), as are the ideal MHD equations. That the expectation value of the helicities is invariant is also shown, for example, by the relation $\left\langle H_{C}\right\rangle=-\partial \ln Z / \partial \beta$, since both $\beta$ and $\left\langle H_{C}\right\rangle$ change sign under $P$ and $C$. This invariance under $P$ and $C$ also requires the expectation values of all odd-order moments of the phase variables to be zero. Yet, when a numerical simulation is run, the time evolution is only over one of the disjoint components, i.e., the theory is non-ergodic.

We can illustrate this disjointness and non-ergodicity pictorially for Case II of Table 1, where $E$ and $H_{C}$ are constants of the motion, but not $H_{M}$. We first transform the phase space variables $\{\tilde{\mathbf{u}}(\mathbf{k}), \tilde{\mathbf{b}}(\mathbf{k}) ; 0<$ $|\mathbf{k}| \leq K\}$ into the equivalent set $\left\{\mathbf{z}^{+}(\mathbf{k}), \mathbf{z}^{-}(\mathbf{k}) ; 0<|\mathbf{k}| \leq K\right\}$, where $\mathbf{z}^{ \pm}(\mathbf{k})=\tilde{\mathbf{u}}(\mathbf{k}) \pm \tilde{\mathbf{b}}(\mathbf{k})$ are the Elsässer variables ${ }^{3}$. Since $E$ and $H_{C}$ are constant, so are the Elsässer energies $E_{1}$ and $E_{2}$ :

$$
E_{1}=E+2 H_{C}=\frac{1}{2}\left[\left|\mathbf{z}^{+}\right|^{2}\right], \quad E_{2}=E-2 H_{C}=\frac{1}{2}\left[\left|\mathbf{z}^{-}\right|^{2}\right] .
$$

Let us assume $H_{C} \geq 0$, so that $E_{1} \geq E_{2}$. The $N_{\Gamma^{-}}$dimensional phase space $\Gamma$ is the direct sum of two $\frac{1}{2} N_{\Gamma^{-}}$ dimensional parts $\Gamma^{+}$and $\Gamma^{-}$, corresponding to the independent transverse parts of the $\mathbf{z}^{+}(\mathbf{k})$ and $\mathbf{z}^{-}(\mathbf{k})$, respectively, with $0<|\mathbf{k}| \leq K$. Since $E_{1}$ is constant, the $\mathbf{z}^{+}(\mathbf{k})$ reside on a hypersphere $S_{1}$ of radius $E_{1}$ in $\Gamma^{+}$, and similarly, the $\mathbf{z}^{-}(\mathbf{k})$ reside on a hypersphere $S_{2}$ of radius $E_{2}$ in $\Gamma^{-}$. The product $T_{12}=S_{1} \times S_{2}$ is represented as the outer torus of Figure 1. Under $P$ or $C, \mathbf{z}^{+}(\mathbf{k}) \rightleftharpoons \pm \mathbf{z}^{-}(\mathbf{k})$, while $\beta \rightarrow-\beta$ and $H_{C} \rightarrow-H_{C}$. The result is that hypersphere $S_{1}$ of radius $E_{1}$ is now in $\Gamma^{-}$, and hypersphere $S_{2}$ of radius $E_{2}$ is in $\Gamma^{+}$. The resulting hypertorus $T_{21}=S_{2} \times S_{1}$ is represented by the inner torus of Figure 1. If $H_{C} \neq 0$, then $T_{12}$ and $T_{21}$ are disjoint and form a nested pair of hypertori, as qualitatively shown in Figure 1. The union $T=T_{12} \cup T_{21}$ is the 'surface of constant energy' and is essentially disjoint ${ }^{9}$ so that the statistical ensemble is non-ergodic ${ }^{19}$. (Figure 1 is also appropriate to Case I of Table 1 with the proviso that the phase point is further constrained to lie on the intersections of the nested hypertori with the hypersurfaces defined by $\pm \mid \bar{H}_{M}$-.)

In contrast to an ensemble prediction arrived at by integrating over all disjoint components labeled by different signs of $H_{C}$ and/or $H_{M}$, when a numerical simulation is run (or a physical system evolves), we start with either the plus or the minus sign of each helicity, but not both. Thus, the symmetry of the theory under $P$ and $C$ is dynamically broken and mean values of the Fourier coefficients with respect to time are not necessarily zero, even though this is the ensemble prediction for ideal MHD turbulence.

Non-ergodicity caused by broken symmetry has been termed 'broken ergodicity' ${ }^{21}$. Numerical examples of this for the various ideal cases in Table 1 have been presented recently ${ }^{13}$, where it was seen that relatively energetic ideal coherent structures arise primarily in Cases I and III (where the invariant magnetic helicity $H_{M}$ had a value of 0.1398). Let us define 'coherent energy' as the energy held in the mean values of the Fourier modes. At the final time step of these previous runs, the coherent magnetic energy was $12.9 \%$ of total energy in Cases I and III, while the coherent kinetic energy was $5.66 \%$ of total energy in Case I and essentially zero for Case III, where $H_{C}$ is not invariant. Less energetic ideal coherent structures occurred and were also seen in Case IV, where $H_{P}$ is invariant. Although the statistical ensemble is manifestly non-ergodic for Case II, there was no energetic coherent structure in that case because unrestricted motion on either hypertori represented in Figure 1 gives each phase variable a time-averaged value of zero. 


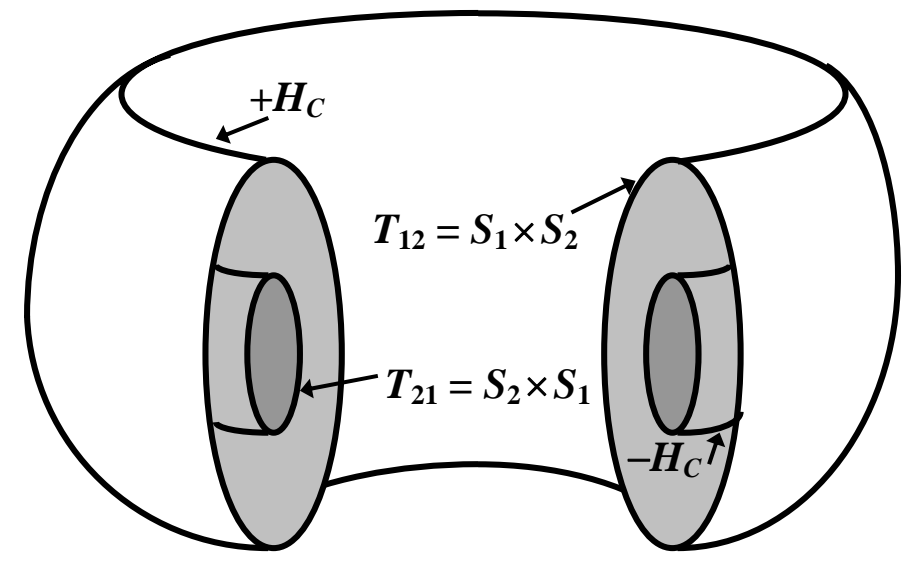

Figure 1: Phase space for ideal MHD turbulence, Case II of Table 1.

In this paper, we study only Cases I and III numerically, and do so for both ideal and real MHD. The reason for this is that only Cases I and III have $\mathbf{B}_{\mathrm{o}}=0$, corresponding to which $H_{M}$ is an ideal invariant. The ideal invariance of $H_{M}$ is evidently required for the appearance of energetic magnetic coherent structure, i.e., for strongly broken symmetry. Our object here is to investigate whether or not this 'magnetic dynamo process' is present in dissipative 3-D MHD turbulence, as it is in ideal cases. The applicability of this work is to understanding the large-scale magnetic fields generated by astrophysical and geophysical objects as a whole, when these objects are situated in negligible external magnetic fields.

\section{Numerical Results}

Here, we present results for real (i.e., decaying) runs, in addition to same-initial condition ideal runs, to see if coherent structure still persists in the presence of dissipation. We move from the $32^{3}$ grid recently used ${ }^{13}$ to a $64^{3}$ grid, which permits a larger dissipation wavenumber while still allowing for relatively long-time simulations on available computer resources.

A Fourier spectral transform method ${ }^{1}$ with $3^{\text {rd }}$-order time-integration ${ }^{22}$ was used for these numerical simulations. Four different $64^{3}$ runs will be discussed. These are the Case I runs: Run 1a $(\nu=\eta=0$, $\left.\Omega_{\mathrm{o}}=B_{\mathrm{o}}=0\right)$ and Run $1\left(\nu=\eta=0.004, \Omega_{\mathrm{o}}=B_{\mathrm{o}}=0\right)$, as well as the rotating Case III runs: Run 1ra $\left(\nu=\eta=0, \Omega_{\mathrm{o}}=1, B_{\mathrm{o}}=0\right)$ and Run $\operatorname{1r}\left(\nu=\eta=0.004, \Omega_{\mathrm{o}}=1, B_{\mathrm{o}}=0\right)$. (Here, 'a' stands for 'absolute equilibrium ensemble', i.e., ideal runs, and ' $\mathrm{r}$ ' stands for 'rotating'.) Runs $1,1 \mathrm{a}, 1 \mathrm{r}$ and 1ra went from $t=0$ to 200 , with time-step $\Delta t=0.001$. For Runs 1 and $1 \mathrm{r}$, the dissipation wavenumber ${ }^{14} K_{D}$ was $K_{D} \approx K$ or less, where $K^{2}=910$.

A note on time units: The dimensionless simulation times quoted here can be related to 'eddy turn-over time' $T_{\Omega} \equiv 2 \pi / \Omega^{1 / 2}$ or 'crossing time' $T_{E} \equiv 2 \pi / E^{1 / 2}$ or some similar unit. In the ideal Runs 1a and 1ra, equilibrium $\Omega \simeq 240$ and $E \simeq 1$, yielding $T_{\Omega} \simeq 0.40$ and $T_{E} \simeq 2 \pi$, so that the dimensionless simulation time unit falls between $T_{\Omega}$ and $T_{E}$. In the dissipative runs, $\Omega \simeq 23$ and $E \simeq 1$ early in the run and decrease to $\Omega \simeq E \simeq 0.003$ at the end; the corresponding $T_{\Omega}$ and $T_{E}$ thus increase from $\sim 1$ to $\sim 100$. These characteristic times may be kept in mind, though we will primarily refer only to dimensionless simulation time $t$.

All these runs had identical initial conditions in which the $\tilde{\mathbf{u}}(\mathbf{k})$ and $\tilde{\mathbf{b}}(\mathbf{k})$ were initialized so that $E_{K}(k) \sim$ $E_{M}(k) \sim k^{4} \exp \left(-2 k^{2} / k_{p}^{2}\right), k_{p}=6$, with random phase. At $t=0$, in all runs, total kinetic energy $E_{K}$ and magnetic energy $E_{M}$ were equal, with $E=E_{K}+E_{M}=1$, while the helicities were $H_{C}=0.34804$ and $H_{M}=0.091968$. During the runs, the constants of the motion drifted by less than $0.1 \%$. The 'constant' values for $E, H_{C}$ and $H_{M}$ are taken to be their time-averages over the ideal runs, and $\left\langle E_{M}\right\rangle$ is found by minimizing the entropy functional (18), which is then used to find $\alpha, \beta$ and $\gamma$ through eq. (16). The results are given in Table 3 . 


\begin{tabular}{cccccccc}
\hline Run & $\bar{E}$ & $\bar{H}_{C}$ & $\bar{H}_{M}$ & $\left\langle E_{M}\right\rangle$ & $\alpha$ & $\beta$ & $\gamma$ \\
\hline 1a & 1.0003 & 0.34794 & 0.091969 & 0.52605 & 1.80134 & -2.38283 & -1.01321 \\
1ra & 1.0006 & 0.00000 & 0.091968 & 0.54627 & 0.96825 & 0.00000 & -0.96810 \\
\hline
\end{tabular}

Table 3: Parameters for Runs 1a and 1ra.

The appearance of coherent structure can be examined directly using phase diagrams, i.e., plots of the real versus the imaginary parts of a Fourier coefficient, which are essentially the projections of the $N_{\Gamma^{-}}$-dimensional phase trajectory onto 2-D planes. Phase diagrams for $\tilde{\omega}_{y}(\mathbf{k}), \tilde{\omega}_{z}(\mathbf{k}), \tilde{b}_{y}(\mathbf{k})$ and $\tilde{b}_{z}(\mathbf{k})$ are presented in Figures 2 and 3 for $\mathbf{k}=(1,0,0)$, and in Figures 4 and 5 for $\mathbf{k}=(2,0,0)$; due to eq. $(4), \tilde{\omega}_{x}(\mathbf{k})=\tilde{b}_{x}(\mathbf{k})=0$ whenever $\mathbf{k}=(k, 0,0)$. (Similar phase diagrams were seen for other $\mathbf{k}$.) Equating 'non-zero mean value' with 'coherent structure', Figure 2 shows that there are both kinetic and magnetic structures for Case I, while Figure 3 shows that there is only magnetic structure for Case III. Additionally, as Figure 6 shows, it is evident that coherent structure exists and is manifested primarily in the $k=1$ modes (as discussed below).

The average energy per x-space grid point is equal to one during the ideal runs, and decreases from an initial value of one for the dissipative runs. The Fourier transforms (1) are defined so that the average corresponding energy per $\mathbf{k}$, with $0<|\mathbf{k}| \leq K$, in k-space is $r^{-1}=2.2733$, where $r$ is defined in (15). The expectation values (14) predict that the $k=1$ modes will have the largest energies, since $\delta^{4}-\alpha^{2} \gamma^{2} / k^{2}$ is smallest for $k=1$ : for Run 1a, $\left\langle|\tilde{\mathbf{u}}(\mathbf{k})|^{2}\right\rangle^{1 / 2}=58.8$ and $\left\langle|\tilde{\mathbf{b}}(\mathbf{k})|^{2}\right\rangle^{1 / 2}=89.0$, while for Run 1ra, $\left\langle|\tilde{\mathbf{u}}(\mathbf{k})|^{2}\right\rangle^{1 / 2}=$ 1.43 and $\left\langle|\tilde{\mathbf{b}}(\mathbf{k})|^{2}\right\rangle^{1 / 2}=88.9$. When $k=2\left(k^{2}=4\right)$, we have $\left\langle|\tilde{\mathbf{u}}(\mathbf{k})|^{2}\right\rangle^{1 / 2}=1.50$ and $\left\langle|\tilde{\mathbf{b}}(\mathbf{k})|^{2}\right\rangle^{1 / 2}=1.62$, while for Run 1ra, $\left\langle|\tilde{\mathbf{u}}(\mathbf{k})|^{2}\right\rangle^{1 / 2}=1.43$ and $\left\langle|\tilde{\mathbf{b}}(\mathbf{k})|^{2}\right\rangle^{1 / 2}=1.66$. There are four independent parts for each of the $\left\langle|\tilde{\mathbf{u}}(\mathbf{k})|^{2}\right\rangle$ and $\left\langle|\tilde{\mathbf{b}}(\mathbf{k})|^{2}\right\rangle$, and while the ensemble prediction is for average equality amongst the respective parts, for $k=1$ this equality is broken because the modes have large mean values, as opposed to the ensemble prediction of zero first-order moments. The values of the modal magnitudes given here are reflected in the ranges of the various axes in Figures 2, 3, 4 and 5.

Ensemble predictions that Fourier modes such as $\tilde{\mathbf{b}}(\mathbf{k})$ are zero-mean random variables does not match the time behavior, particularly for $k=1$, and this is shown in Figures 2 to 5 . Non-ergodicity (due to the broken symmetry) is indicated by the non-zero mean values that are very large compared to the associated standard deviations; in the very-long time $32^{3}$ ideal runs recently reported ${ }^{13}$, this ratio was as high as 18 , and is similar here. After a brief initial transient period, the phase point arrives at an attractor: for Runs 1 and $1 \mathrm{a}$, Figure 2 shows that the ideal trajectory arrives at its attractor at $t \approx 70$, while the real trajectory peaks at $t \approx 52$ (the phase path ends at $t=200$ ).

Although the location of the ideal attractor depends on initial conditions, the equations of motion and statistical theory are invariant under $P C T$, as well as under galilean transformations $(\mathbf{x} \rightarrow \mathbf{x}+\mathbf{a})$ and rotation of the coordinate system. Thus, the attractor can be placed in various equivalent locations and, in effect, an ensemble prediction does just this, leading to a prediction of zero mean values for the first-order moments. Also, please remember that non-ergodicity results from disjointness ${ }^{19}$, and exists whether or not non-zero mean values of first-order moments occur.

It is evident that the ideal and real modes in Figures $2-5$ track each other fairly closely initially, and are qualitatively similar over the whole trajectory. This indicates the pertinence of ideal results to real turbulent flows, particularly at low values of $k$. Here, this is evident on $64^{3}$ grids, where $\nu=\eta=0.004$, and should be far more evident when larger grids are used, with correspondingly smaller $\nu$ and $\eta$. However, there is always a trade-off between grid-size and run-time, so that small grid-sizes are needed if long run-times are desired, for example, in statistical studies.

Also note that Figures 2 to 5 show what appears to be a general result, termed 'depression of nonlinearity $^{\prime 23}$, i.e., the tendency to a force-free state ${ }^{24}$. In eqs. (2) and (3), the nonlinear effects are due to $\mathbf{u} \times \boldsymbol{\omega}$, $\mathbf{j} \times \mathbf{b}$ and $\mathbf{u} \times \mathbf{b}$; these vector cross products become small when $\mathbf{u} \sim \boldsymbol{\omega}, \mathbf{j} \sim \mathbf{b}$ and $\mathbf{u} \sim \mathbf{b}$. For modes with $\mathbf{k}=(k, 0,0)$, this translates to $\tilde{\omega}_{y}(\mathbf{k}) \sim \pm i \tilde{\omega}_{z}(\mathbf{k}), \tilde{b}_{y}(\mathbf{k}) \sim \pm i \tilde{b}_{z}(\mathbf{k})$ and $\tilde{\omega}_{y}(\mathbf{k}) \sim \pm i \tilde{b}_{z}(\mathbf{k})$, respectively. In other words, depression of nonlinearity manifests itself for $\mathbf{k}=(k, 0,0)$ modes as a $\pm \pi / 2$ phase shift of $\tilde{\omega}_{y}(\mathbf{k})$ and $\tilde{b}_{y}(\mathbf{k})$ with regard to $\tilde{\omega}_{z}(\mathbf{k})$ and $\tilde{b}_{z}(\mathbf{k})$, respectively. If $\mathbf{u} \sim \mathbf{b}$, we also expect $\tilde{\omega}_{y}(\mathbf{k}) \sim \pm \tilde{b}_{y}(\mathbf{k})$ and 
(a) $\omega_{\mathrm{y}}(1,0,0), 1 \mathrm{a}$ : Black, 1: Gray

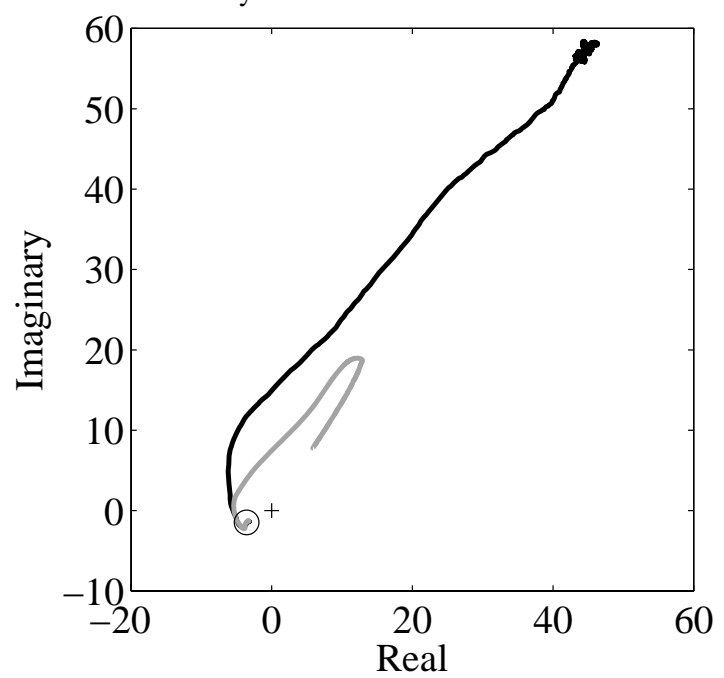

(c) $b_{y}(1,0,0), 1 a:$ Black, 1: Gray

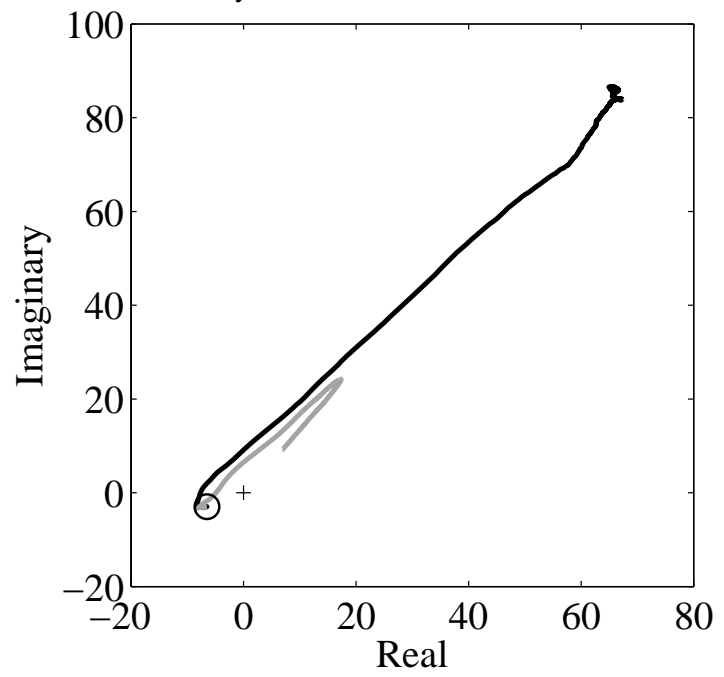

(b) $\omega_{z}(1,0,0), 1 \mathrm{a}$ : Black, 1: Gray

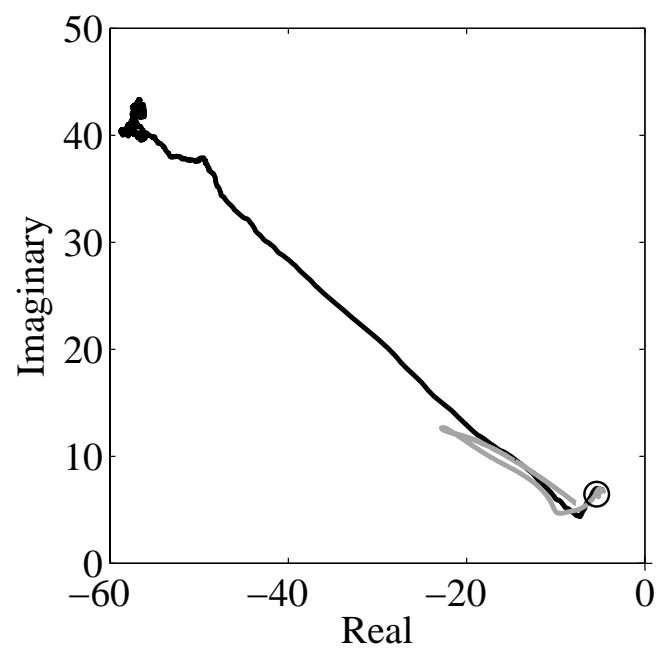

(d) $b_{z}(1,0,0), 1 a:$ Black, 1: Gray

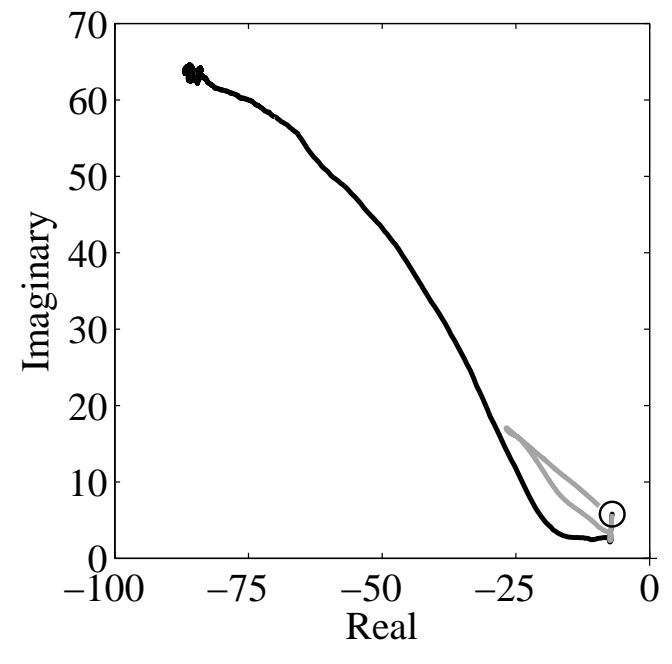

Figure 2: Phase portraits for the $\mathbf{k}=(1,0,0)$ modes of Runs 1 and 1a. $\bigcirc$ indicates $t=0$. 
(a) $\omega_{y}(1,0,0), 1$ ra: Black, 1r: Gray

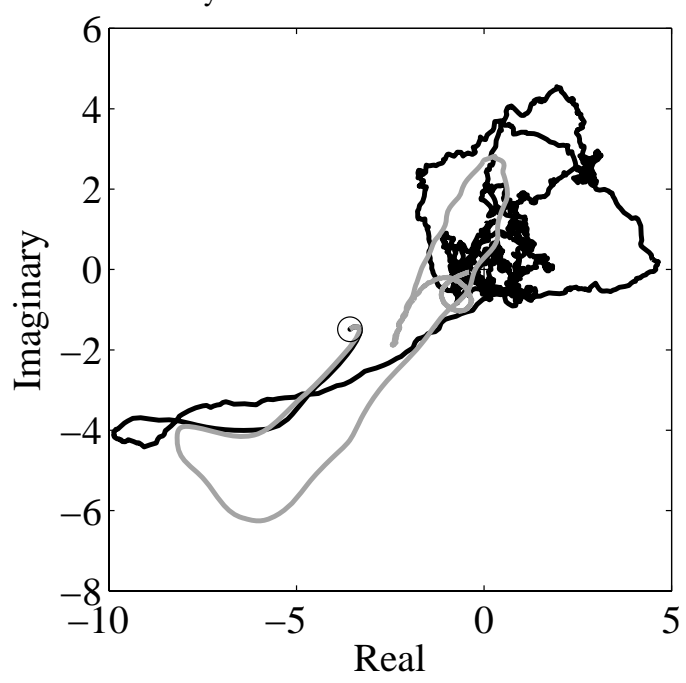

(c) b $(1,0,0)$, 1ra: Black, 1r: Gray

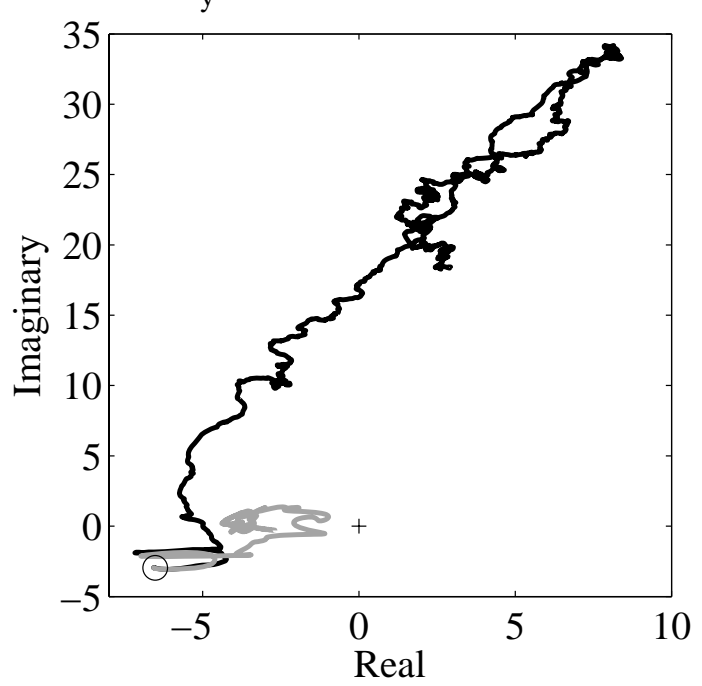

(b) $\omega_{z}(1,0,0)$, 1ra: Black, 1r: Gray

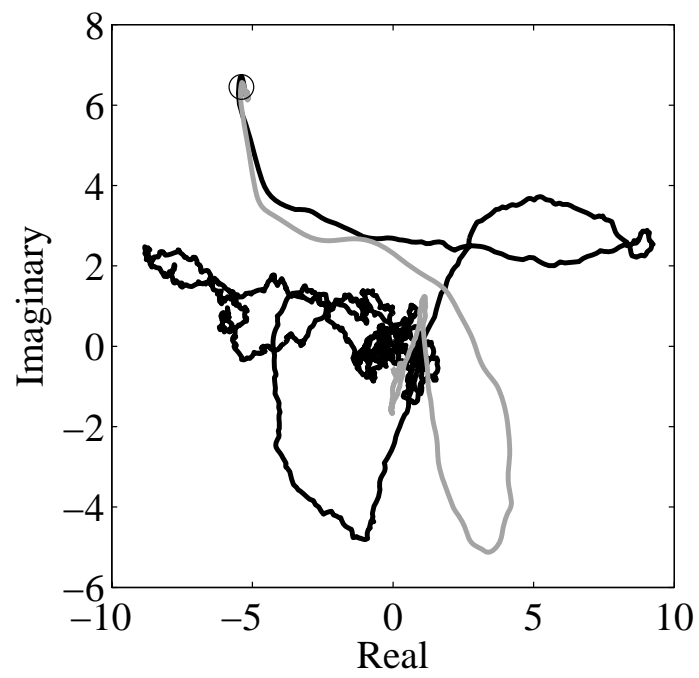

(d) $b_{z}(1,0,0)$, 1ra: Black, 1r: Gray

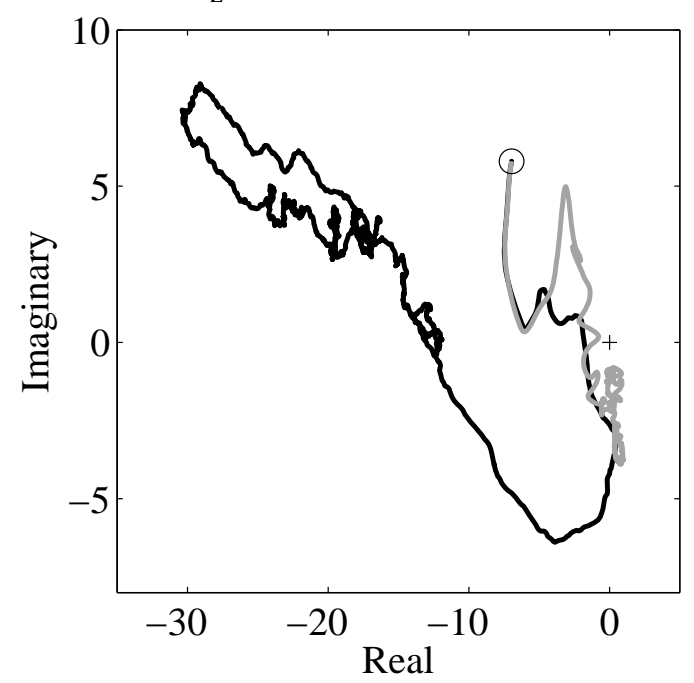

Figure 3: Phase portraits for the $\mathbf{k}=(1,0,0)$ modes of Runs $1 \mathrm{r}$ and 1ra. $\bigcirc$ indicates $t=0$. 
(a) $\omega_{\mathrm{y}}(2,0,0), 1 \mathrm{a}$ : Black, 1: Gray

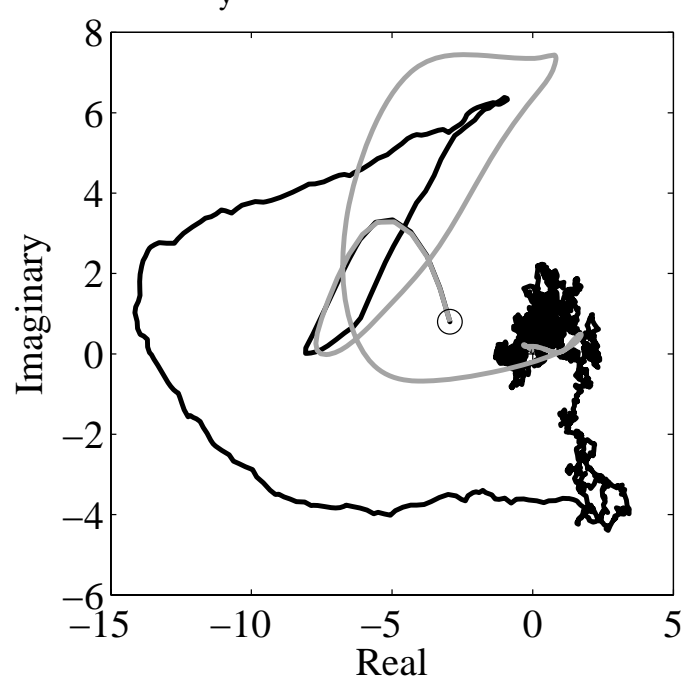

(a) $\mathrm{b}_{\mathrm{y}}(2,0,0)$, 1a: Black, 1: Gray

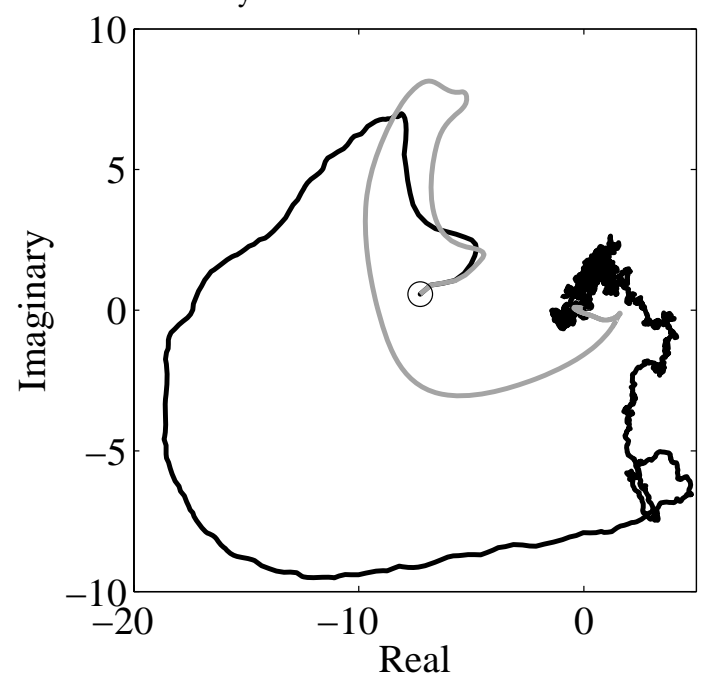

(a) $\omega_{z}(2,0,0), 1 \mathrm{a}:$ Black, 1: Gray

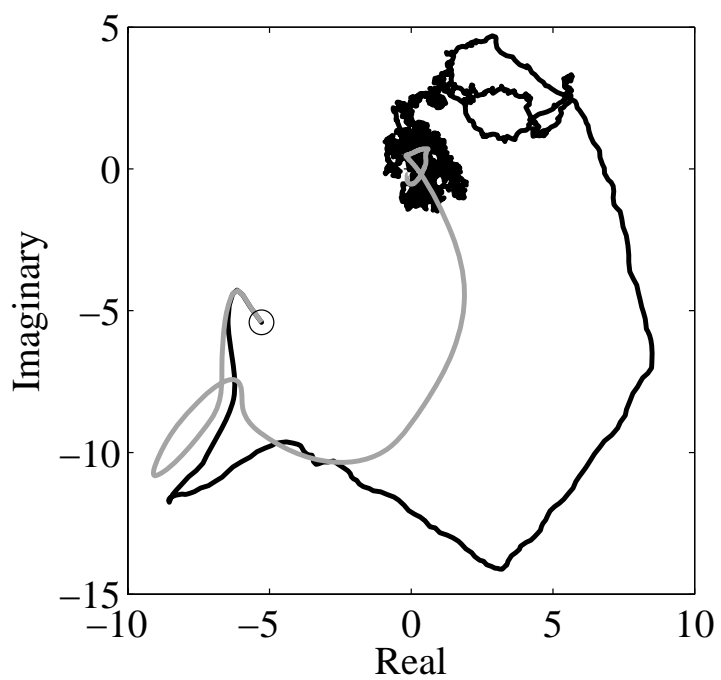

(a) $b_{z}(2,0,0), 1 a:$ Black, 1: Gray

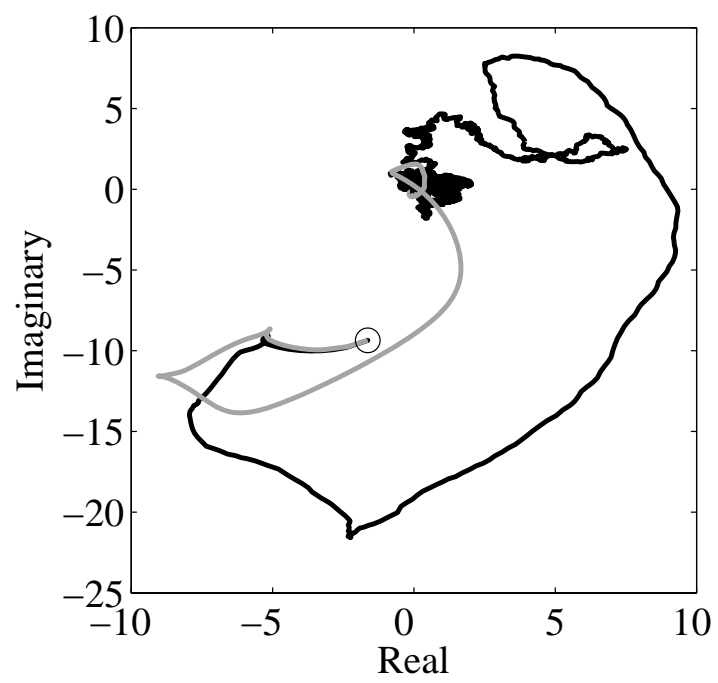

Figure 4: Phase portraits for the $\mathbf{k}=(2,0,0)$ modes of Runs 1 and $1 \mathrm{a}$. $\bigcirc$ indicates $t=0$. 

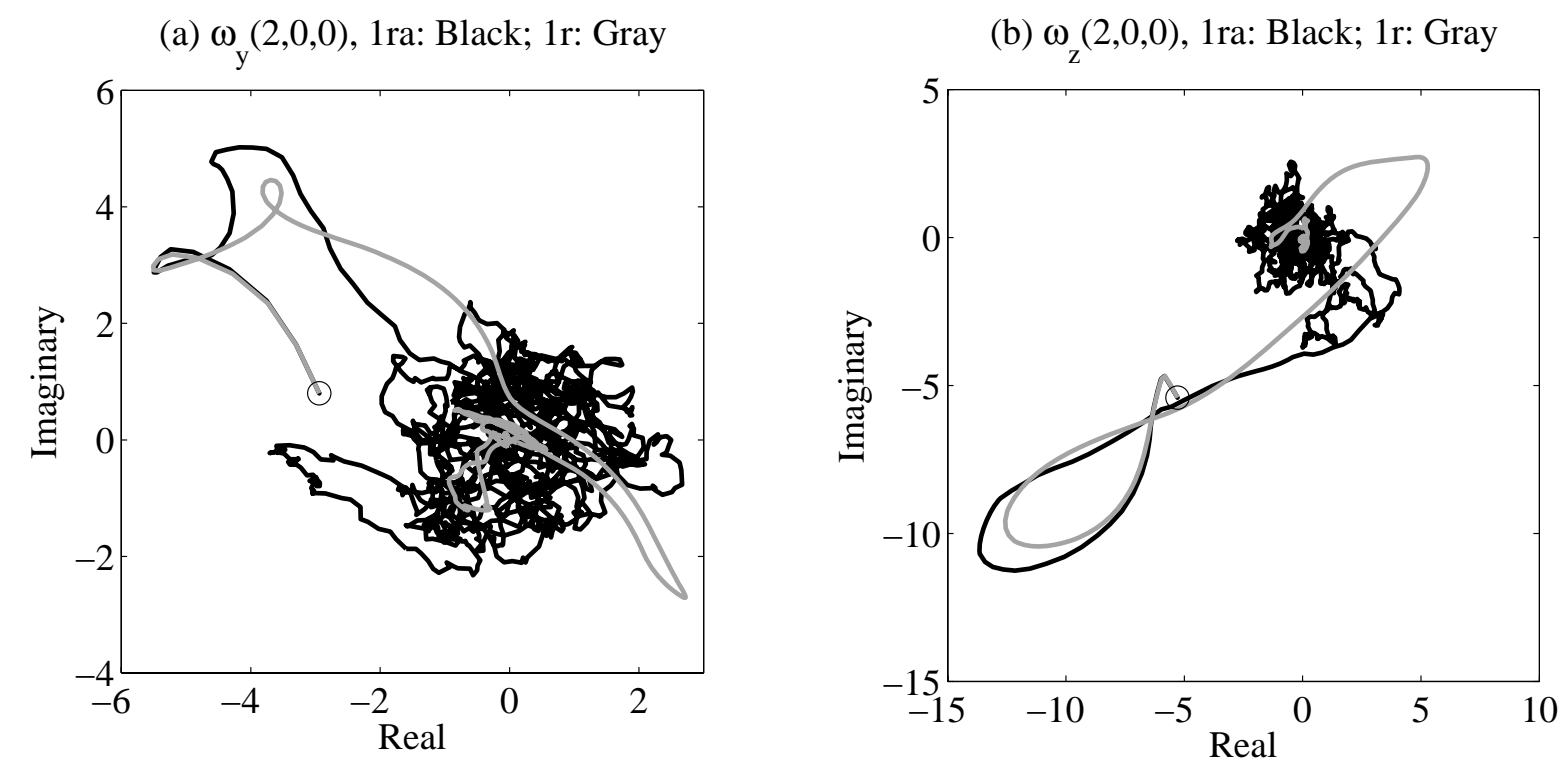

(c) $b_{y}(2,0,0)$, 1ra: Black; 1r: Gray

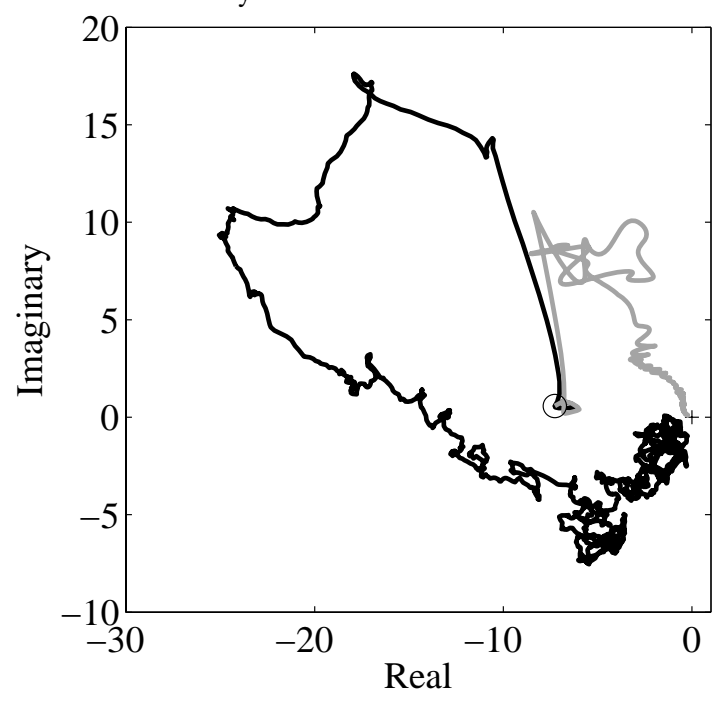

(d) $b_{z}(2,0,0), 1$ ra: Black; 1r: Gray

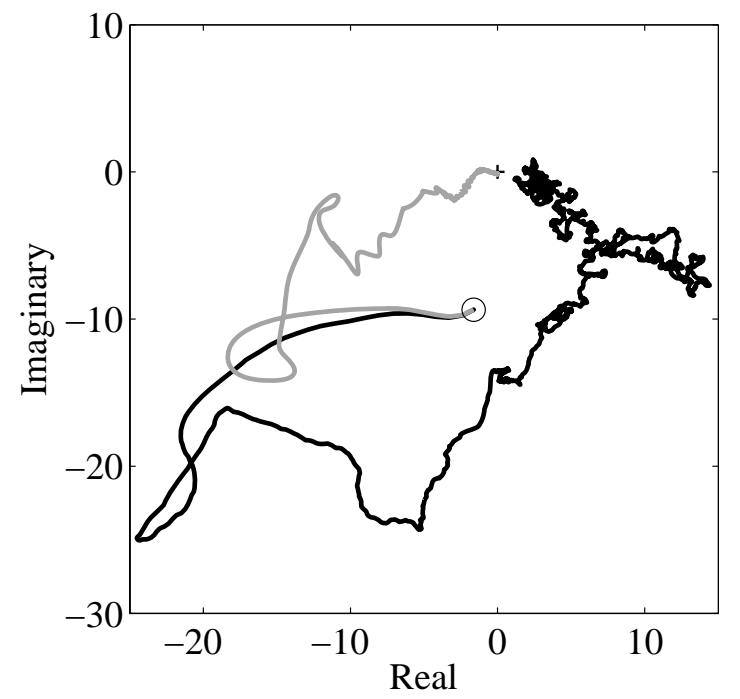

Figure 5: Phase portraits for the $\mathbf{k}=(2,0,0)$ modes of Runs 1r and 1ra. $\bigcirc$ indicates $t=0$. 
$\tilde{\omega}_{z}(\mathbf{k}) \sim \pm \tilde{b}_{z}(\mathbf{k})$. These relationships are clearly seen in Figures $2-5$. (Since we are dealing with a statistical ensemble, we may also view 'depression of nonlinearity' as an application of Le Chatelier's principle ${ }^{25}$.) As nonlinearity is depressed, characteristic times associated with nonlinear evolution are increased, essentially reducing the usefulness of qualitative temporal measures such as the previously discussed $T_{\Omega}$ and $T_{E}$.

In Figure 6, the time evolution of energy in modes with $k^{2}=1,2,3$ and 4, as well as the total energy for all modes, for the various Runs are shown. In particular, the transfer of energy between shells of different $k$ is apparent; initially the spectrum was peaked at $k^{2} \approx 6$, and Figure 6 shows that it follows an 'inverse cascade $^{5}$ to smaller and smaller values of $k$. In the ideal Runs 1a and 1ra, Figure 6 (a) and (c) show that equilibrium is almost established by $t=200$. In the decaying Runs 1 and 1r, Figure 6 (b) and (d) also show an inverse cascade, even though the total energy is monotonically decaying: For Run 1, the energy in the $k^{2}=4,3,2$ and 1 modes peaks at $t \approx 2,10,20$ and 50, respectively, while for Run $1 \mathrm{r}$, the behavior is similar, except that the $k^{2}=1$ energy profile is flatter and peaks slightly at $t \approx 70$. Additionally, the $k^{2}=2$ modal energy appears to be dominant for a longer time in Run 1ra and 1r, as Figure 6 (b) and (d) show. These rotating runs also dissipate energy more slowly than the non-rotating runs; this decrease in dissipation for rotating turbulence has been previously noted ${ }^{26}$. Overall, Figure 6 shows that the inverse cascade, coupled with the attractor evident in Figures 2-5, indicates the growth of a coherent structure or magnetic dynamo.

Figure 6 indicates that ideal Runs 1a and 1ra have much of their final energy held in the $k=1$ modes. In fact, most of this $k=1$ energy is in the associated coherent structures. In Run 1a, the coherent magnetic energy is $7.83 \%$ and coherent kinetic energy is $3.59 \%$ of total energy at $t=200$, while in Run 1ra, the coherent magnetic energy is $6.26 \%$ and coherent kinetic energy $\sim 0 \%$ of total energy at $t=200$. These percentages are similar to what was seen previously ${ }^{13}$ for Case I and III runs. In regard to the decaying Runs 1 and 1r, Figures 2 and 3 show that the $k=1$ modes behave in a very coherent manner, while Figure 6 shows that the energy in dissipative Runs 1 and $1 \mathrm{r}$ is essentially all in the $k=1$ modes at $t=200$. Thus, the dissipative runs appear to evolve so that almost $100 \%$ of their energy in a coherent, albeit decaying, structure.

Please note that Figures 6 (a) and (c) show that the ideal turbulence simulations are not quite yet in equilibrium. Using eqs. (14), the energy in the $k^{2}=1$ modes is predicted to be 0.1302 for Run 1a and 0.09051 for Run 1ra. In comparison, the (fluctuating) data associated with Figures 6 (a) and (c) have reasonably close values of 0.1296 and 0.08774 , respectively, at $t=200$. However, for $k^{2}=2$, the corresponding equilibrium values for Runs 1a and 1ra are 0.0001553 and 0.0001418 , while the values at $t=200$ are 0.001107 and 0.003071 , respectively. Thus, while the $k=1$ modes have essentially arrived at their equilibrium values, for $k>1$ this requires some more time, as is indicated in the general downward slope of the $k^{2}=2,3$ and 4 modes in Figures 6 (a) and (c). However, the attainment of complete equilibrium is not our goal here, as it was in recent $32^{3}$ simulations $^{13}$, where the run-time went from $t=0$ to 2000 , so that an equilibrium spectrum for all $k$ was achieved.

Next, we consider the evolution of the cross helicity $H_{C}$ and the magnetic helicity $H_{M}$ for the different runs. In Figure 7, corresponding to the non-rotating Runs 1 and 1a, we see that total (i.e., summed over all modes) $H_{C}$ and $H_{M}$ are conserved for Run 1a, as they should be. For the ideal Run 1a, the difference in $H_{C}$ and $H_{M}$ is that the contribution of the $k=1$ modes is only about $15 \%$ to the total $H_{C}$, while the contribution of the $k=1$ modes to $H_{M}$ is essentially $100 \%$. This is the inverse cascade of magnetic helicity observed long ago ${ }^{5}$. For the decaying Run 1, Figure 7 shows that all of the value of $H_{C}$ and $H_{M}$ is concentrated in the $k=1$ modes at the end of the runs. Furthermore, we note that the cusps in Figure 7 are related to a change in sign of the $k=1$ parts of $H_{C}$ and $H_{M}$, in that these are negative before the cusp and positive afterwards.

In the rotating Runs $1 \mathrm{r}$ and $1 \mathrm{ra}, H_{C}$ is not an ideal invariant, while $H_{M}$ remains so. This is clearly seen in Figure 8 (a), where the multitude of cusps indicate that the total $H_{C}$ and its $k=1$ part are both fluctuating about zero. Figure 8 (b), however, shows that $H_{M}$ in the rotating Runs $1 \mathrm{r}$ and 1a behave very similarly to $H_{M}$ in the non-rotating runs, by comparison with Figure 7 (b). In all runs, ideal and real, magnetic helicity concentrates itself in the largest scale $(k=1)$ available. Thus, the ideal invariance of $H_{M}$, which is the common feature between Cases I and III of Table 1, appears essential for the creation of coherent structure in both ideal and dissipative MHD turbulence, with or without rotation.

In fact, the concentration of magnetic helicity is more important in the decaying runs, than in the ideal ones, because the 'normalized helicities' actually grow in value. Here, the normalization of cross and magnetic 
(a) Run 1a

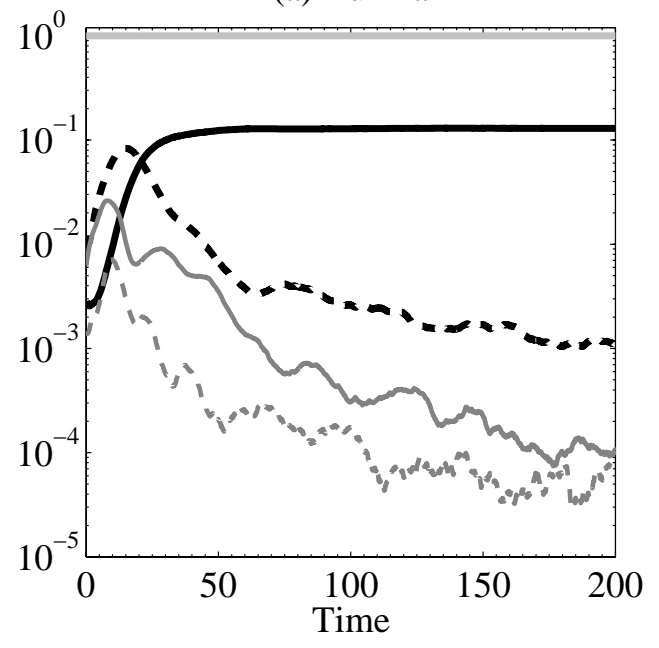

(c) Run 1ra

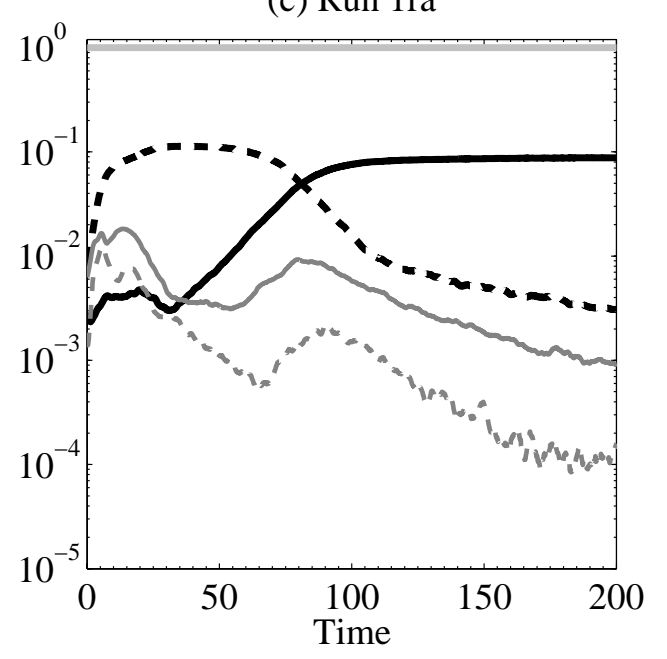

(b) Run 1

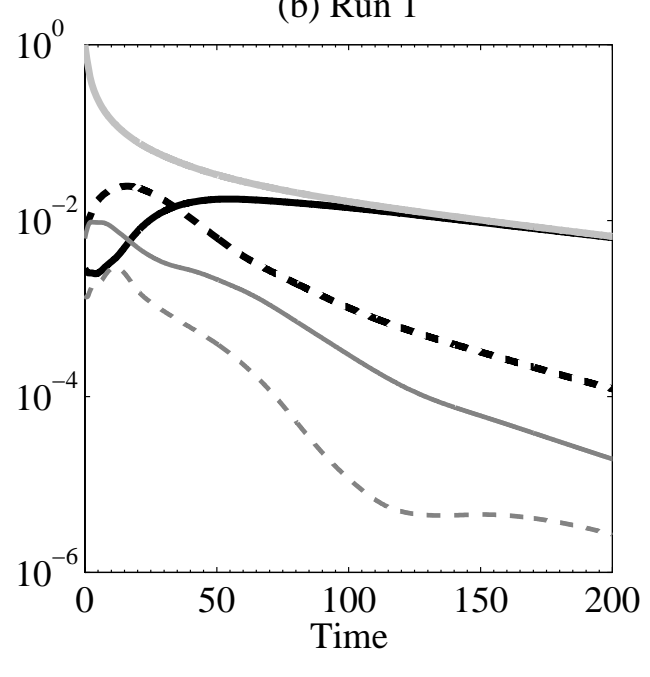

(d) Run $1 \mathrm{r}$

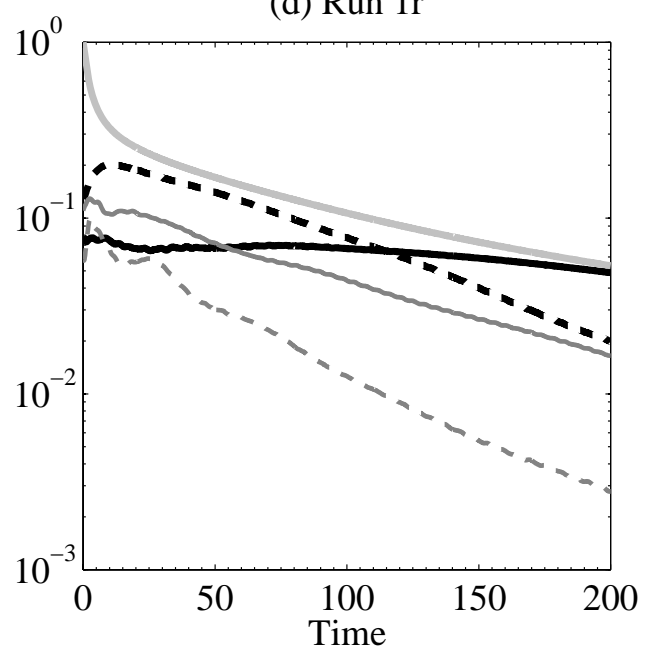

Figure 6: Energy in modes with given $k^{2} \leq 4$ vs time; $E_{t o t}$ is the energy in all modes. 

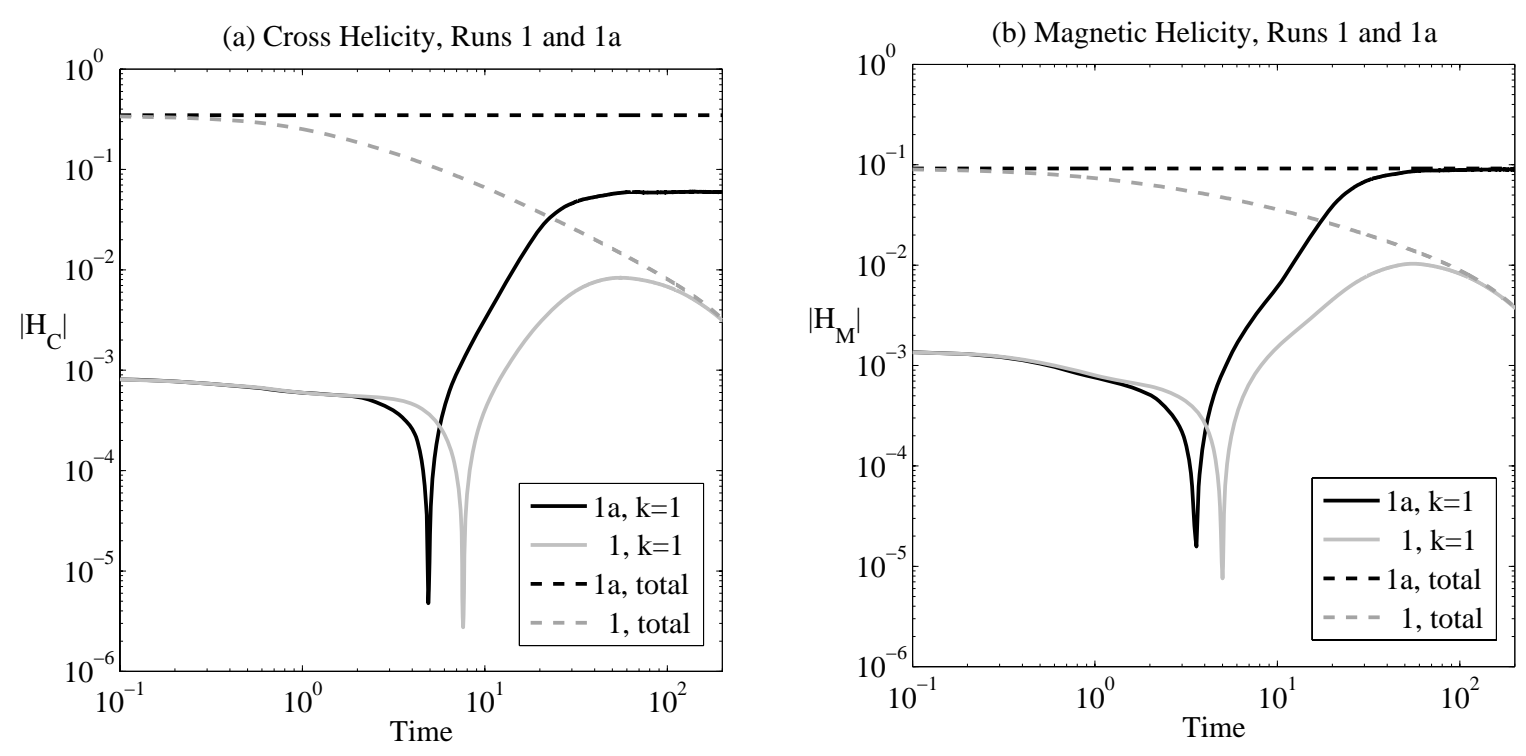

Figure 7: Time evolution of $\left|H_{C}\right|$ and $\left|H_{M}\right|$ for Runs 1 and 1a.
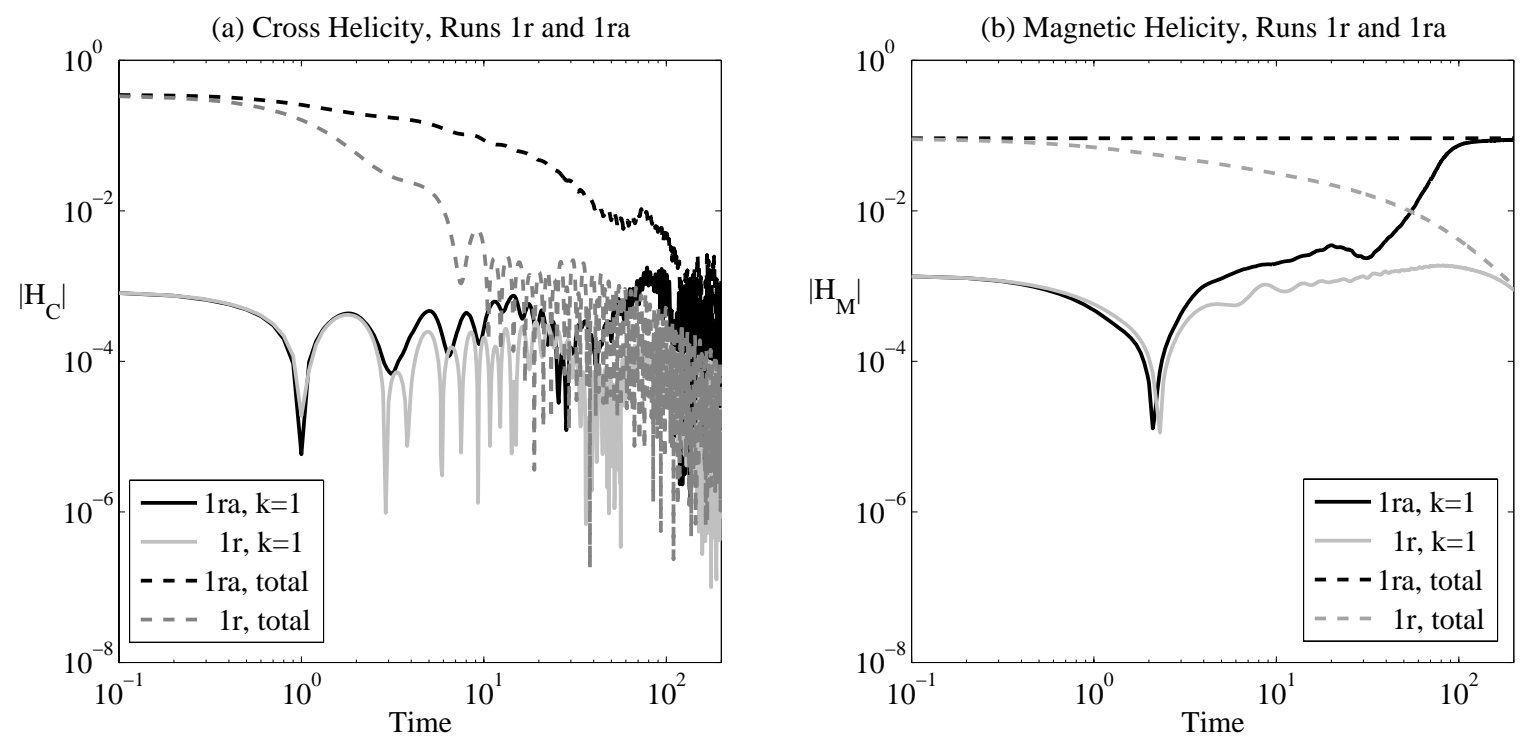

Figure 8: Time evolution of $\left|H_{C}\right|$ and $\left|H_{M}\right|$ for Runs 1r and 1ra. 
(a) Runs 1a and 1ra

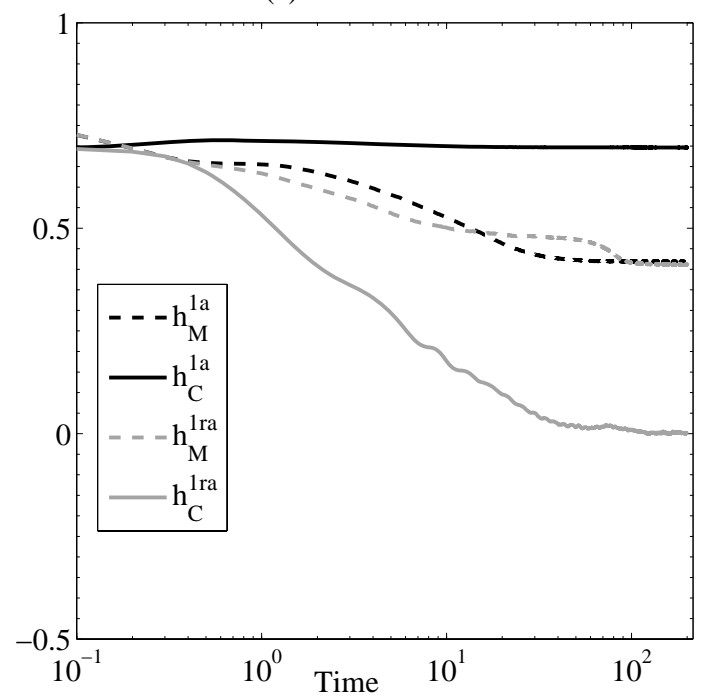

(b) Runs 1 and $1 \mathrm{r}$

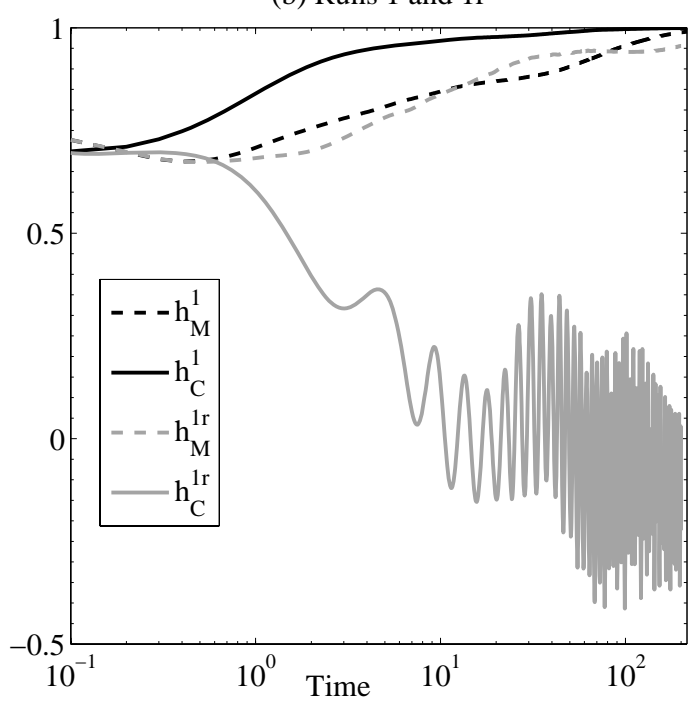

Figure 9: Normalized helicities for (a) Runs 1 and 1a; (b) Runs 1r and 1ra.

helicities will be defined in the following manner:

$$
h_{C} \equiv \frac{H_{C}}{\left(E_{K} E_{M}\right)^{1 / 2}}, \quad h_{M} \equiv \frac{H_{M}}{\left(E_{M} A\right)^{1 / 2}} .
$$

Above, we use $E_{K}=\frac{1}{2}\left[u^{2}\right], E_{M}=\frac{1}{2}\left[b^{2}\right]$ and $A=\frac{1}{2}\left[a^{2}\right]$.

In Figure 9 (a), the normalized helicities for the ideal Runs 1a and 1ra are presented, while in Figure 9 (b), the normalized helicities for the dissipative Runs 1 and $1 \mathrm{r}$ are given. Although dissipation leads to overall energy decay, it is clear in Figure 9 that if $H_{C}$ and/or $H_{M}$ are invariant in the ideal runs, they decay less quickly than energy in the corresponding dissipative runs. This is the process of 'selective decay' ${ }^{27,28,29,30}$, by which states of dynamically aligned or anti-aligned $\mathbf{u}, \boldsymbol{\omega}$ and $\mathbf{b}$ arise.

In fact, 'dynamical alignment' and 'selective decay' seem equivalent to 'depression of nonlinearity'. Broken symmetry, on the other hand, allows for large mean values of the $k=1$ modes. Coupled with 'selective decay', it leads, as we have seen, to relatively energetic coherent structures. In particular, it leads to large coherent magnetic structures in Cases I and III of Table 1.

\section{Conclusion}

Although the simulations discussed were on a relatively small grid of $64^{3}$ points, this allowed for runs from $t=0$ to 200 (there is always this trade-off in numerical simulations). In the ideal Run 1a, broken symmetry and non-ergodicity ('broken ergodicity' ${ }^{21}$ ) leads to coherent kinetic and magnetic structures similar to that found in recent $32^{3}$ runs $^{13}$ that evolved from $t=0$ to 2000 . In the present work, the real Run 1 had a $k=1$ modal trajectory close to that of ideal Run $1 \mathrm{a}$, until about $t \approx 50$. Similar behavior was observed in the rotating Runs 1ra and 1r, with the difference that only magnetic coherent structure developed.

These structures manifest themselves at the lowest wave numbers $k=1$, that is, at the largest length scales of the system, where eqs. (14) predict the largest ideal modal energies. These structures are due to ideal invariants, specifically to the pseudoscalars $H_{C}$ and $H_{M}$, which enter into eqs. (14) through the pseudoscalar inverse temperatures $\beta$ and $\gamma$ via eqs. (16). As we have seen, the ensemble phase space has disjoint components identified by plus and minus signs of the invariant helicities, which allows for the symmetry of the phase space to be maintained under the discrete transformations $C$ and $P$.

Although ensemble averages are taken over all components in phase space, dynamical evolution is confined to only one component, thereby breaking the symmetry inherent in the phase space and ensuring the canonical 
ensemble is not ergodic. This non-ergodicity is often readily apparent in the non-equivalence of ensemble and time averages; however, there are cases where the dynamical system is non-ergodic but where ensemble and time averages happen to agree, at least for first and second-order moments. Perhaps this non-ergodicity can be seen in the study of third or higher-order moments, or through some other measure.

We expect that lower dissipation runs on larger grids will produce even more slowly decaying, longerlived and energetic magnetic dynamos. These larger grids will also allow for a clearer view of modal energy transfer and modal contribution to emerging coherent structures. Of course, grid sizes cannot be too large if very long simulations times are desired. Studies of forced turbulence should also prove interesting, but care must be taken so as not to introduce artifacts due only to the particular forcing method chosen. (Boussinesq convection is a natural method of forcing, but this moves us away from the five cases listed in Table 1, since a new physical field, temperature variation, is introduced.)

In addition to continuing the study of homogeneous turbulence using Fourier series, investigations with other spectral methods, for example using spherical harmonic expansions, may also be very informative. It is a straightforward matter to keep track of the means, variances, and higher-order moments of the expansion coefficients, whether the spectral method of choice uses sines and cosines, or spherical harmonics. Spherical harmonic methods (e.g., with Chebyshev expansions in the radial direction) are not uncommon, but statistical studies of the associated canonical ensemble of coefficients appear to be absent.

In summary, what we have seen here using Fourier spectral methods and relatively small grids is the persistence in dissipative flows of the effects of broken symmetry and non-ergodicity that were first seen in ideal simulations. The result of this 'broken ergodicity' in MHD turbulence is that coherent structure appears to grow out of initially random and unstructured initial conditions. The most energetic structures occur in Cases I and III of Table 1, and these seem to be primarily magnetic in nature. This 'magnetic dynamo process' is due to the topology of phase space, which is a collection of disjoint sets. Rotation does not itself cause this, and the strength of the magnetic structures does not appear to be greatly affected by rotation (it is only the kinetic part of the structure that disappears when rotation is present). In fact, studies of magnetic stars indicate only a 'loose ... correlation between magnetic flux and angular rotational velocity' $^{31}$.

Of course, the solar interior and the earth's outer core are not incompressible, homogeneous magnetofluids, so that compressibility and other effects must eventually be taken into account. Here, for now, we must content ourselves with some interesting and suggestive results found in the investigation of incompressible, homogeneous MHD turbulence. These results probably follow more from the presence of pseudoscalar helical invariants in MHD turbulence, and less from the theoretical approximations employed or numerical methods used. This essential feature may help these results retain their pertinence when more detailed physical models and numerical methods are implemented.

\section{References}

1. G. S. Patterson and S. A. Orszag, Phys. Fluids 14, 2538 (1971).

2. T. D. Lee, Q. Appl. Math. 10, 69 (1952).

3. W. M. Elsässer, Rev. Mod. Phys. 28135 (1956).

4. L. Woltjer, Proc. Nat. Acad. Sci. USA 44, 489 (1958).

5. U. Frisch, A. Pouquet, J. Leorat, and A. Mazure, J. Fluid Mech. 68, 769 (1975).

6. J. V. Shebalin, Ph.D. thesis, College of William and Mary (1982).

7. J. V. Shebalin, Physica D 37, 173 (1989).

8. J. V. Shebalin, Phys. Plasmas 1, 541 (1994).

9. J. V. Shebalin, Phys. Lett. A 250, 319 (1998).

10. A. Pouquet and G. S. Patterson, J. Fluid Mech. 85, 305 (1978).

11. M. Meneguzzi, U. Frisch and A. Pouquet, Phys. Rev. Lett. 47, 1060 (1981). 
12. R. S. Miller, F. Mashayek, V. Adumitroaie, and P. Givi, Phys. Plasmas 3, 3304 (1996).

13. J. V. Shebalin, J. Plasma Phys. 72, 507 (2006).

14. D. Biskamp, Magnetohydrodynamic Turbulence (Cambridge University Press, UK, 2003).

15. J. V. Shebalin, NASA TP-2002-210783, 2002.

16. J. V. Shebalin, "Broken symmetry and coherent structure in MHD turbulence," in MPA/P15: Proceedings of the Workshop on Interdisciplinary Aspects of Turbulence (Ringberg Castle, Tegernsee, Germany, 18-22 April 2005) edited by F. Kupka and W. Hillebrandt (Max-Planck-Institut für Astrophysik, Garching, 2005), pp. 77-92.

17. T. D. Lee and C. N. Yang, Phys. Rev. 104254 (1956).

18. T. Stribling and W. H. Matthaeus, Phys. Fluids B 2, 1979 (1990).

19. A. I. Khinchin, Mathematical Foundations of Statistical Mechanics (Dover, New York, 1949), pp. 137-145.

20. J. V. Shebalin, J. Plasma Phys. 56, 419 (1996).

21. R. G. Palmer, Adv. Phys. 31, 669 (1982).

22. J. Gadzag, J. Comp. Phys. 20, 196 (1976).

23. R. H. Kraichnan and R. Panda, Phys. Fluids 31, 2395 (1988).

24. J. B. Taylor, Phys. Rev. Lett. 33, 1139 (1974).

25. L. D. Landau \& E. M. Lifshitz, Statistical Physics (Pergamon, New York, 1980), 3rd Ed., §22.

26. J. Bardina, J. H. Ferziger and R. S. Rogallo, J. Fluid Mech. 154, 321 (1985).

27. M. Dobrowolny, M. Mangeney, and P. Veltri, Phys. Rev. Lett. 45, 144 (1980).

28. W. H. Matthaeus and D. Montgomery, Ann. N.Y. Acad. Sci. 357, 203 (1980).

29. J. P. Dahlburg, D. Montgomery, G. D. Doolen, and L. Turner, Phys. Rev. Lett. 57, 428 (1986).

30. T. Stribling and W. H. Matthaeus, Phys. Fluids B 3, 1848 (1991).

31. S. Hubrig, P. North, and G. Mathys, Astrophys. J. 539, 352 (2000). 